\title{
Laboratorio de Nuevos Materiales Nanoestructurados y Catálisis Heterogénea

\author{
División de Materiales Avanzados, \\ Instituto Potosino de Investigación Científica y Tecnológica, A.C.
}

\author{
Vicente Rodríguez-González*
}

\begin{abstract}
RESUMEN: El Laboratorio de Nuevos Materiales Nanoestructurados y Catálisis Heterogénea (LANOCAT) fue creado el año 2010 dentro de la División de Materiales Avanzados (DMAV) del Instituto Potosino de Investigación Científica y Tecnológica A.C. (IPICyT). Actualmente es un laboratorio consolidado que apoya a estudiantes e investigadores a nivel regional, nacional e internacional vía colaboraciones y/o proyectos de investigación. En este documento se informa de la infraestructura del laboratorio y se describen las actividades de investigación que ahí se realizan: síntesis de nanomateriales, caracterización estructural y estudio de superficies activas, así como las respectivas aplicaciones en nanociencias y nanotecnología con énfasis en catálisis heterogénea. Se presenta una breve reseña del IPICyT, del posgrado en nanociencias y materiales de la DMAv perteneciente al Programa Nacional de Posgrados de Calidad del Consejo Nacional de Ciencia Tecnología (PNPC-CONACyT) así como de las líneas de investigación cultivadas en esta división. Finalmente se mencionan los laboratorios nacionales con que cuenta el IPIcyт que complementan y apoyan de manera importante la investigación de frontera. Se describen las funciones de la Dirección de Vinculación, instancia encargada de apoyar la trasferencia de la ciencia básica para su aplicación con el objetivo de generar desarrollo tecnológico y promover la innovación y así coadyuvar al crecimiento científico y tecnológico sostenible y a la internacionalización del Instituto y de la ciencia en México.
\end{abstract}

PALABRAS CLAVE: Nanomateriales, nanociencias, sostenible, catálisis heterogénea, IPIcyt, química de superficies, LINAN.

ABSTRACT: The Nanostructured New Materials and Heterogeneous Catalysis Laboratory (LANOCAT, for its acronym in Spanish) was created in 2010 in the Advanced Materials Department of The Institute for Scientific and Technological Research of San Luis Potosi, (IPICyT, for its acronym in Spanish). During the seven years of activity, the equipment of this laboratory give support to national and international researchers and students via collaborations or research grants. In this document, the laboratory infrastructure is described with the research areas such as nanomaterials synthesis, materials characterization, surface science and the nanoscience and nanotechnology applications focusing on heterogeneous catalysis. A brief introduction about IPICyT, the Nanoscience and Materials Graduate Program of the Advanced Materials Department which is recognized as of high quality by the National Quality Graduate Program of the National Council of Science and Technology (PNPC-CONACyT, for its acronym in Spanish). In addition the research lines cultivated in the Department will be briefly described. Finally, the national labora-

Recibido: 23 de enero de 2017. Aceptado: 4 de abril de 2017.

* Posgrado en Nanociencias y Materiales-División de Materiales Avanzados. Camino a la Presa de San José No. 2055, Edificio Beta. Lomas 4ta Sección, 78216, San Luis Potosí, sLP, México. IPICyт A.C. Tel (444)834 2000 ext.7295, Fax (444) 834 2010. Correspondencia: (catalisisbeta@ipicyt.edu.mx); (vicente.rdz@ipicyt.edu.mx); (linan@ipicyt.edu.mx). 
Mundo Nano | CATÁLISIS EN MÉxICo | www.mundonano.unam.mx

10(18), enero-junio 2017 | DOI: 10.22201/ceiich.24485691e.2017.18.60108

tories that have been established at the IPICyT are outlined. The latter give strong support to perform original and cutting edge research together with the activities of Innovation and Knowledge Transfer Department which is also described because it helps the transfer process from fundamental science towards technological development and innovation; this contributes to sustainable scientific, technological growth and to the internationalization of our Institute and science in Mexico.

KEYWORDS: Nanomaterials, nanoscience, sustainable, heterogeneous catalysis, IPICyT, surface science, LINAN.

\section{Introducción}

El Instituto Potosino de Investigación Científica y Tecnológica A.C., (IPICyT) es una asociación civil fundada por el gobierno del estado de San Luis Potosí, el Consejo Potosino de Ciencia y Tecnología, la Universidad Autónoma de San Luis Potosí, la Secretaría de Educación Pública, el Consejo Nacional de Ciencia y Tecnología, el Centro de Investigación en Matemáticas, A.C. y el Centro de Investigación en Materiales Avanzados, S.C.

Es un centro público de investigación multi e interdisciplinario del sistema CONACyT fundado el 24 de noviembre del año 2000, y representa un esfuerzo importante en pro de la descentralización de las actividades científicas y tecnológicas en el país. Su fundación fue también posible gracias al interés y apoyo explícito del gobierno del estado de San Luís Potosí, quien aportó recursos para la construcción de los primeros edificios que albergan al Instituto (www.ipicyt.edu.mx).

Fue establecido con el propósito de proveer a la región de un espacio alternativo para el cultivo de las ciencias naturales y exactas, así como para desarrollar tecnologías vinculadas con la solución de problemas locales, regionales y nacionales. El Instituto considera también entre sus objetivos estratégicos la difusión y transferencia del conocimiento generado por sus actividades de investigación, así como la formación de recursos humanos de excelencia en campos del conocimiento de frontera.

El IPICyT cuenta con grupos de investigación de alta calidad que, además de generar conocimientos de frontera y formar recursos humanos a nivel licenciatura y posgrado, interaccionan con los diversos sectores de la sociedad para apoyar el desarrollo del San Luís Potosí y la región centro del país.

La investigación se realiza en los siguientes departamentos:

- Biología Molecular <http://www.ipicyt.edu.mx/Biologia_Molecular/areas_biologia_ molecular.php $>$

- Materiales Avanzados <http://www.ipicyt.edu.mx/Materiales_Avanzados/areas_materiales_ avanzados.php> 

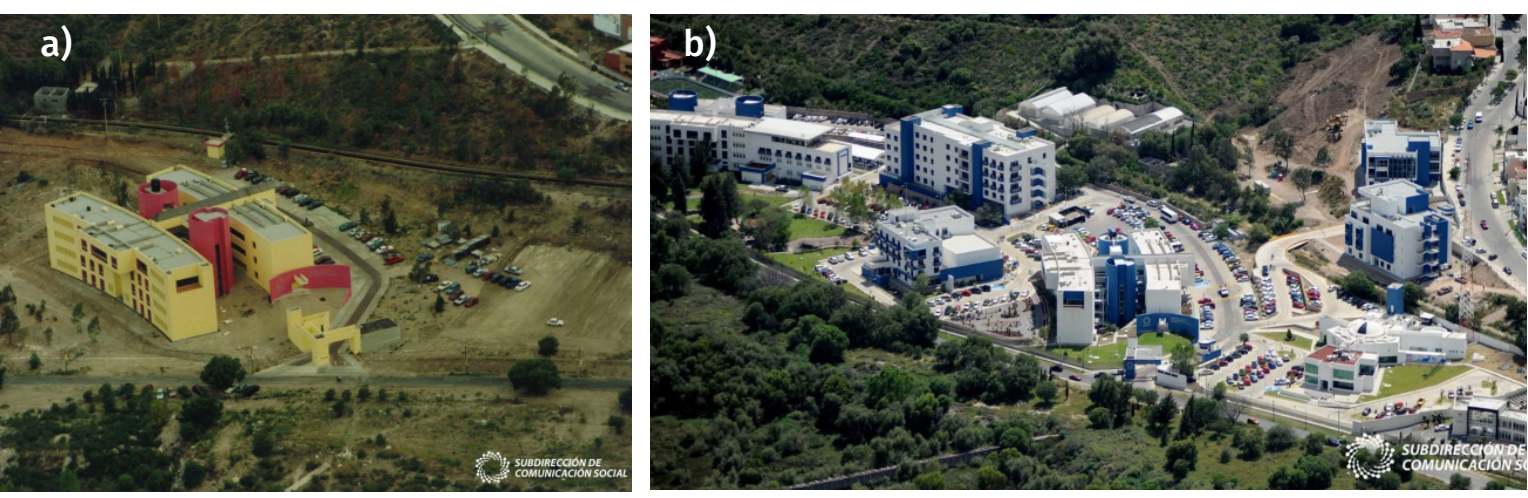

FIGURA 1. Fotografía de los edificios con que cuenta del IPIcyт desde su creación (a) hasta la última imagen tomada en 2016 (b). A la izquierda de la imagen b) se pude ver el edificio beta con dos torres donde se alberga la División de Materiales Avanzados y el Laboratorio de Nuevos Materiales y Catálisis Heterogénea.

- $\quad$ Matemáticas Aplicadas <http://www.ipicyt.edu.mx/Matematicas_Aplicadas/areas_ matematicas_aplicadas.php>

- Ciencias Ambientales

<http://www.ipicyt.edu.mx/Ciencias_Ambientales/areas_ciencias_ ambientales.php>

\section{- Geociencias Aplicadas}

<http://www.ipicyt.edu.mx/Geociencias_Aplicadas/areas_geociencias_ aplicadas.php $>$

Cabe destacar que una de las características distintivas del Instituto es abordar problemas de investigación de manera interdisciplinaria en las áreas de ciencias naturales y exactas. Aunque su compromiso inmediato está ligado al estado de San Luís Potosí, su misión ciertamente le permite trascender la región y tener cabida en los ámbitos nacional e internacional, debido a la excelencia de sus productos de investigación y a la oportunidad existente para los proyectos estratégicos que desarrolla en las áreas de conocimiento de su ámbito de competencia.

Para estos propósitos, cuenta con tres Laboratorios Nacionales y una Dirección de Vinculación:

- $\quad$ lanbama (Laboratorio Nacional de Biotecnología Agrícola, Médica y Ambiental)

<http://www.ipicyt.edu.mx/Lanbama/lanbama.php>

- LINAN (Laboratorio Nacional de Investigaciones en Nanociencias y 
Nanotecnología)

$<$ http://www.linan-ipicyt.mx/>

- Laboratorio Nacional del cNs (Centro Nacional de Supercómputo) $\langle$ http://www.ipicyt.edu.mx/Cns/cns.php〉

La infraestructura de los Laboratorios Nacionales así como los servicios que ofrecen, se pueden consultar en la página del instituto (www.ipicyt.edu. $\mathrm{mx}$ ) o en los sitios web de cada División.

\section{La Dirección de Vinculación}

〈http://www.ipicyt.edu.mx/Vinculacion/vinculacion.php〉

Fue creada para construir puentes entre los investigadores y los sectores productivos y gubernamentales, y con el propósito de transferir conocimiento, hacer desarrollo tecnológico y promover la innovación para crear un impacto real en la sociedad. De igual manera, provee servicios relacionados con la vigilancia y protección tecnológica. Sus funciones se detallan en su sitio web.

\section{División de Materiales Avanzados (DMAv)}

El desarrollo de nuevos materiales nanoestructurados y sus crecientes aplicaciones en los últimos años ha impulsado las investigaciones en materiales y nanotecnología en todo el mundo. En la División estamos convencidos de que el descubrimiento de nuevas tecnologías debe estar basado en la comprensión de los conceptos científicos básicos, los cuales a su vez, darán pautas para proponer conceptos nuevos que puedan establecer el conocimiento de frontera.

En esta división se realiza la síntesis, caracterización y el empleo de nuevos materiales y nanoestructuras para su uso en aplicaciones emergentes. Nanomateriales como grafeno, titanias, nanotubos, nanocompósitos de carbono, materiales híbridos y biomateriales diversos. Asimismo, materiales magnéticos a base de diferentes aleaciones con propiedades calóricas con miras a utilizarse en aplicarse en aplicaciones tales como la refrigeración de estado sólido, generación de fuentes sustentables de energía, electrónica orgánica, sensores de gases. Finalmente, materiales para nanomedicina, inactivación de microrganismos patógenos, entre otros.

La investigación en el área de catálisis heterogénea y ciencia de materiales, tanto en el área de aplicación como de síntesis de nuevos materiales, se concentra en la DMAv y se agrupa en las siguientes líneas de investigación:

(i) Materiales y compuestos nanoestructurados: fotocatalisis, nanocompósitos, producción de hidrógeno, electrónica orgánica, sensores de gases.

(ii) Materiales magnéticos: refrigeración electromagnética, aleaciones con memoria de forma 
(iii) Biomateriales y bionanotecnología: biofuncionalización, biofísica molecular, inactivación de microrganismos, nanomedicina.

El área de investigación en nanomateriales aplicados a catálisis heterogénea y nanotecnología se desarrolla generalmente en el Laboratorio de Nuevos Materiales y Catálisis Heterogénea (LNMyCH) con importante apoyo del LINAN. Las diversas investigaciones de frontera se enfocan en:

i) El desarrollo de nuevos materiales nanoestructurados con propiedades catalíticas y semiconductoras (nanocompositos grafeno-óxidos metálicos, óxidos con crecimiento preferenciales, nanoestructuras facetadas, NPs metálicas de $\mathrm{Au}, \mathrm{Ni}, \mathrm{Ag}, \mathrm{Cu}, \mathrm{Pd}$, etc.). Materiales mesoporosos ordenados de $\mathrm{SiO}_{2}, \mathrm{TiO}_{2}$ y $\mathrm{Al}_{2} \mathrm{O}_{3}$.

ii) Caracterizaciones generalmente enfocadas en la determinación y cuantificación de sitios activos superficiales así como para interpretación con espectroscopías FTIR y UV-vis-NIR in-situ en función de atmósfera y temperatura, determinación de planos expuestos y crecimiento preferencial en nanocatalizadores y materiales con morfología controlada 1D-3D por microcopías electrónicas y espectroscopía fotoelectrónica de rayos X.

iii) Las aplicaciones son diversas: procesos de oxidación avanzada, reacciones catalíticas inducidas a baja temperatura e inactivación de microrganismos patógenos, degradación solar y fotoasistida de disruptores endócrinos y fármacos, así como también para materiales para la dosificación controlada de fármacos y producción fotocatalítica de hidrógeno.

\section{Infraestructura del LANOCAT}

La infraestructura del Laboratorio de Nuevos Materiales Nanoestructurados y Catálisis Heterogénea se enumera a continuación:

i) Síntesis de nanomateriales.

- Reactor hidrotérmico asistido por microondas, Eyela MWO-1000 Wave Magic capacidad $150 \mathrm{~mL}$.

- Reactores pyrex para síntesis sol-gel diseño propio del grupo de investigación, con sistema de reflujo y calentamiento homogéneo.

- Sistema de fotodeposición de nanopartículas metálicas de $\mathrm{Ag}, \mathrm{Cu}$, $\mathrm{Co}, \mathrm{Sn}$ etc. sobre diferentes estructuras de óxidos mixtos y grafeno.

- Sistema para síntesis fotoasitida de nanopartículas metálicas con lámparas LEDs a diferentes longitudes de onda y UV.

- Sistema de síntesis pyrex de nanoalambres por el método poliol con reflujo y adición controlada.

- Spin Coater Model KW-4A, Two Stage Spinning, $110 / 60 \mathrm{~Hz}$ para el 
Mundo Nano | CATÁLISIS EN MÉXICo | www.mundonano.unam.mx

10(18), enero-junio 2017 | DOI: 10.22201/ceiich.24485691e.2017.18.60108

depósito de películas delgadas de óxidos mixtos y nanoestructuras de carbono.

- Depósito de recubrimientos de óxidos mixtos y materiales de carbono por aerografía y doctor-blade.

- Sistemas de filtrado a vacío para neutralización y lavado de óxidos mixtos y grafeno.

- Hornos de secado, FELISA, para secado hasta $250{ }^{\circ} \mathrm{C}$, y sistemas de secado a vacío hasta $200^{\circ} \mathrm{C}$.
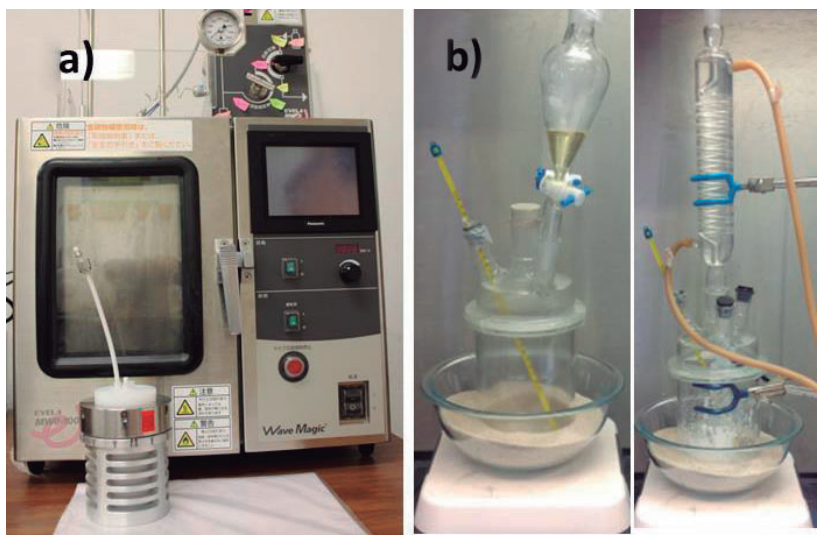

FIGURA 2. Equipos y accesorios para síntesis de nanomateriales: a) reactor de microondas Eyela mwo1000 Wave Magic; b) reactor de síntesis sol-gel con adición controlada y sistema de condensación.

FIGURA 3. a) Reactor de síntesis por métodos foto-inducidos; b) estufas de secado al vacío y mufla de calcinación a $1000^{\circ} \mathrm{C}$; c) mufla de calcinación hasta $1200^{\circ} \mathrm{C}$.

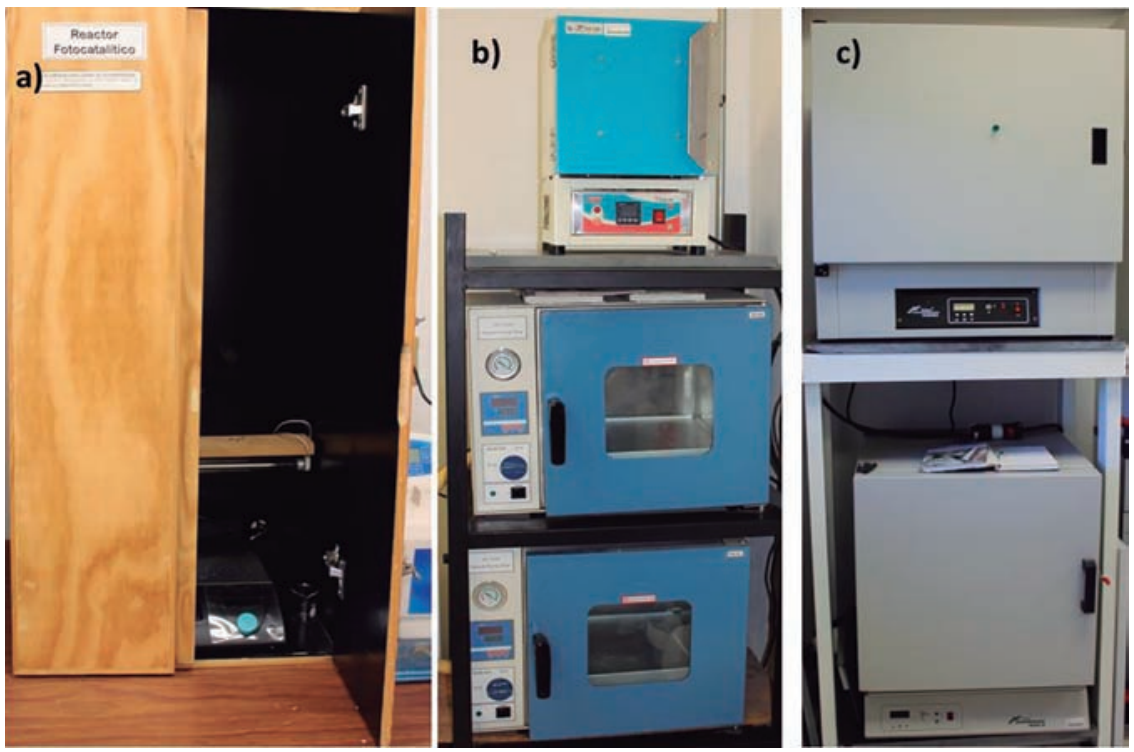


- Muflas para calcinación hasta 1000 y $1200{ }^{\circ} \mathrm{C}$ (con 5 rampas para tratamientos térmicos controlados).

ii) Caracterización

- Espectrofotómetro UV-vis-NIR equipado con esfera de integración (Agilent Cary 5000) con accesorios para líquidos, sólidos, y películas delgadas y cámara Praying mantis de reacción para estudios con atmósfera controlada y temperatura (DR-UV-vis).

- Equipo de fisisorción de nitrógeno Quantachrome Nova3200e para área superficial específica y determinación de tipo de mesoporosidad.

- Equipo de quimisorción de gases en función de la temperatura, (TPR, TPO,TPD) ChemBET de Quantachrome.

- Medidor de carbono orgánico total -TOC-L de Shimadzu, mide hasta ppm de COT.

- Espectrofotómetro infrarrojo con trasformada de Fourier, sólidos, líquidos y reactor Praying Mantis para DRIFTS y con una cámara de reacción con modulación de temperatura de Harrick, IR-Tracer-100 de Shimadzu.

- Potenciostato/Galvanostato modelo VSP300 con chasis modular

FIGURA 4. Equipos de caracterización fisicoquimica: a) fisisorción de nitrógeno; b) quimisorción de gases sonda; c) potenciostato/galvanostato, y, d) determinador de carbón orgánico disuleto.
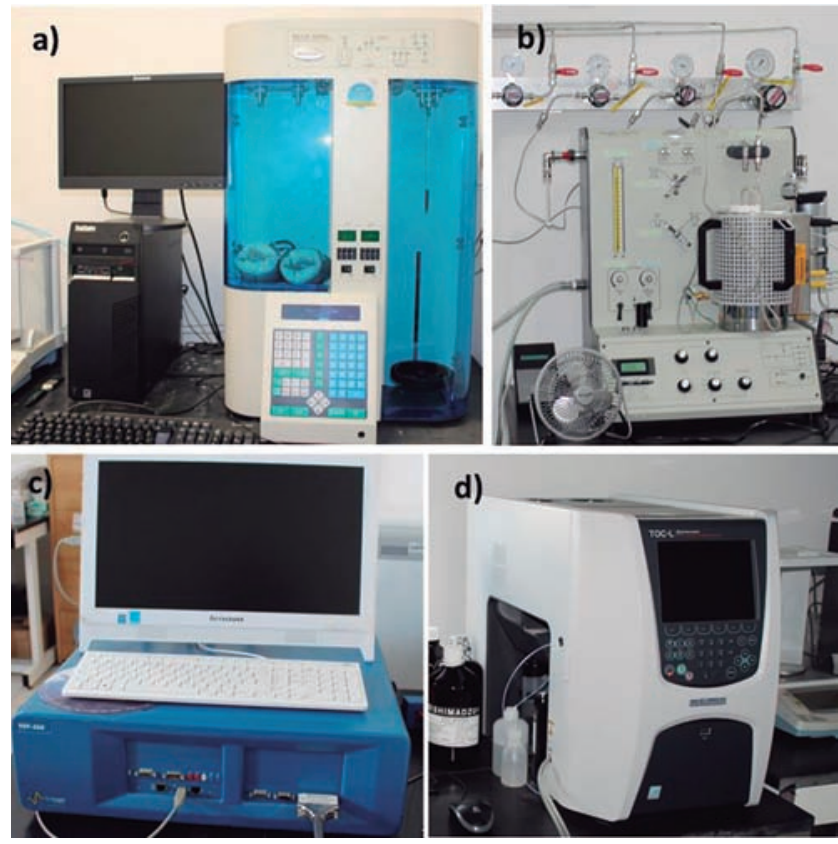
Mundo Nano | CATÁLISIS EN MÉXICo | www.mundonano.unam.mx

10(18), enero-junio 2017 | DOI: 10.22201/ceiich.24485691e.2017.18.60108
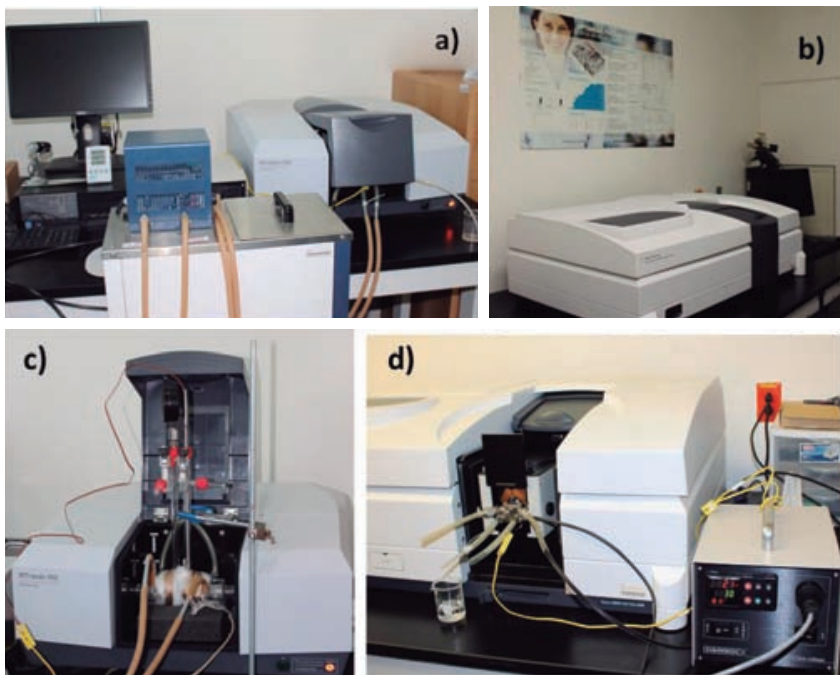

FIGURA 5. Espectrofotómetros: a la izquierda (a) Infrarrojo con trasformada de Fourier, y, la derecha (b) Ultravioleta-visible con Infrarrojo cercano, ambos equipados con esfera de reflactancia difusa y cámara praying para caracterización in situ en función de la temperatura o atmósfera controlada (c) y adsorción de piridina (d).

para 6 slots independientes VSP-300 Bio-Logic Science Instruments SAS.

- $\quad$ Fuente de voltaje-corriente (Keithley 4200) de cuatro puntas.

iii) Evaluación

- Cromatógrafo de gases GC-TCD (Thermo Scientific, TRACE GC Ultra) equipado con una columna empacada TG-BOND Msieve 5A, para monitoreo de la producción de hidrógeno.

- Cromatógrafo de gases GC-FID (Thermo Scientific, TRACE GC Ultra) equipado con una columna empacada PONA, para la evaluación de reformación de hidrocarburos.

- Cromatógrafo de gases GC-MS (Agilent, 5977 Series GC/MS Systems).

- HPLC cromatógrafo de líquidos de alta eficiencia con fotodetector y detector de aniones y cationes.

- 2 reactores de evaluación fotocatalítica con aislante de fuentes luminosas de madera, con diferentes irradiaciones UV, visible y LED, agitación y burbujeo de oxígeno.

\section{Integrantes del laboratorio}

El investigador responsable de LANOTEC es el autor de este trabajo, quien realiza el trabajo del laboratorio en colaboración con posdoctorantes (vía convocatoria del CONACYT) y estudiantes de doctorado, maestría y licen- 

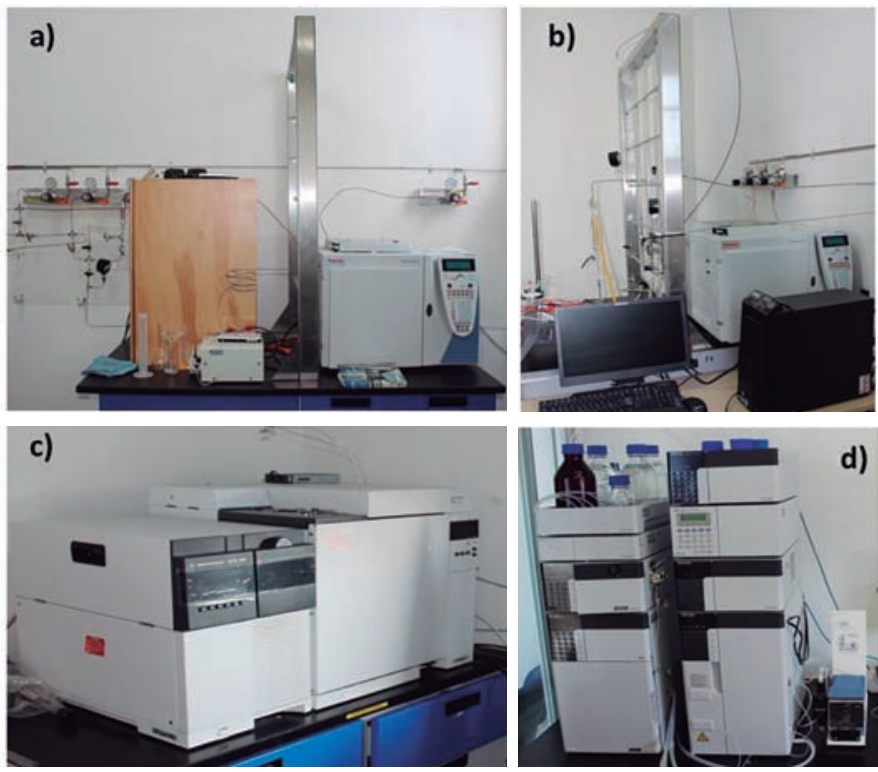

FIGURA 6. Cromatógrafos para evaluación de la reacciones catalíticas y productos: a) producción fotocatalitica de hidrógeno; b) reformación de hidrocarburos; c) GC-MS para estudio de los posibles mecanismos y productos, y, d) HPLC aniónico-catiónico.

ciatura, el promedio anual varía entre 5 y 10 colaboradores. Además, el laboratorio es sede para estudiantes del "Verano de la Investigación Científica”. El apoyo técnico especializado lo brinda fundamentalmente el Laboratorio Nacional de Investigaciones en Nanociencias y Nanotecnología (LINAN).

\section{Posgrado en Nanociencias y Materiales}

El Posgrado de Nanociencias y Materiales está adscrito a la DMAv y cuenta con las modalidades de maestría y doctorado. Además apoya la realización de tesis de licenciatura de diferentes universidades e institutos nacionales e internacionales, así como estancias de veranos de los programas existentes en el país (prácticas profesionales, servicio social, cotutorias, colaboraciones). El posgrado fue evaluado en 2011, obteniendo el PNPC-CONACyT y refrendándolo en 2014, para 2017 se contempla obtener el PNPC internacional.

Para la realización de sus actividades el posgrado tiene acceso a todos los equipos que conforman el Laboratorio Nacional de Investigaciones de Nanociencias y Nanotecnología que cuenta con una de las mayores infraestructuras a nivel de Laboratorios Nacionales enfocado a servicios de análisis y caracterización de materiales de alta calidad para el desarrollo de la nanociencia y la nanotecnología en México. El posgrado también utiliza las instalaciones del Centro Nacional de Supercómputo, para llevar a cabo simulaciones mole- 
culares que tienen el objetivo de entender una gran variedad de propiedades fisicoquímicas, electrónicas y magnéticas.

Una de nuestras fuerzas radica en las diversas colaboraciones internacionales con que contamos, así como la gran movilidad de nuestros estudiantes que realizan estancias en EUA, Canadá, Latinoamérica, Asia y Europa. Por tanto, las opciones de nuestro posgrado tienen carácter multidisciplinario e internacional.

La misión de la DMAv es ofrecer un posgrado multidisciplinario, líder en la formación de recursos humanos de calidad internacional en el área de $\mathrm{Na}-$ nociencias y Materiales. Que nuestros egresados cuenten con prestigio, reconocimiento social, y sean capaces de adaptarse a nuevos retos. Asimismo, los egresados se distingan por su independencia, liderazgo, compromiso social y competitividad en el desarrollo científico en general y nanotecnológico en particular.

Formar recursos humanos en áreas estratégicas de innovación y prioritarias en México para los sectores privado, gubernamental y académico.

Fortalecer la investigación de vanguardia mediante la incorporación de estudiantes a proyectos estratégicos de la institución. Alcanzar una vinculación óptima con los sectores públicos y privados mediante la capacitación y actualización continua del cuerpo académico, la innovación y el fortalecimiento de la infraestructura. Con ello, convertirnos en un polo científico nacional con reconocimiento internacional en las áreas de materiales y nanotecnología.

\section{Productos académicos y formación de estudiantes}

Desde la creación del grupo de Nuevos Materiales Nanoesctruturados y Catálisis Heterogénea del IPICyT en 2010 se han producido 40 publicaciones internacionales indizadas, graduado 5 estudiantes de doctorado, 5 de maestría 12 de licenciatura de diferentes regiones del país y de Colombia, se ha atendido a cerca de 45 veranos de la ciencia y estancias (becas mixtas, servicio social, estancias técnicas, etc.).

Cuatro proyectos han hecho posible la consolidación del grupo, proyectos de la convocatorias innova, ciencia básica, infraestructura y de cooperación con Corea del Sur y España financiados por sus gobiernos, intercambios académicos con la Universidad de Cincinnati, la Universidad de Bogotá y la Universidad Industrial de Santander, entre otras.

El grupo ha participado en la organización de los congresos internacionales: 2nd. Latin-American Congress of Photocatalysis and $\mathrm{TiO}_{2}$ ( $\mathrm{LACP}$ 2013) celebrado en Guadalajara en septiembre de 2013, y, 3rd Latin-American Congress of Photocatalysis, Photoelectrochemical and Photobiology, realizado éxitosamente en las instalciones del IPICyT en octubre de 2014 (3er-LACP-2014), dando pie a 3 números especiales en las revistas indizadas internacionalmente: Journal Hazardous Materials (FI 5.12), Catalysis Today 
(FI 3.42) Journal Environmental Chemical Engineering (nuevo en 2013). Actualmente se mantienen las siguientes colaboraciones internacionales y nacionales:

- Universidad Sun Moon, Corea del Sur.

- University of Cincinnati, USA.

- Loughborough University, UK.

- Universidad Autónoma de Madrid.

- Universidad Industrial de Santander.

- Universidad de Cartagena.

- Universidad de Bogotá-Fundación Tadeo Lozano.

- Universidad Autónoma Metropolitana-Iztapalapa.

- cCAdET-UnAM.

- IF-UNAM.

- Universidad de Zacatecas.

- Universidad. Guanajuato.

- Universidad Autónoma de San Luis Potosí.

- IIM-UNAM.

- Divisiones de Biología Molecular y Ciencias Ambientales del ipicyT.

\section{Referencias y apoyos}

1. Sitio institucional:

$\langle$ www.ipicyt.edu.mx>.

2. Sección especial:

Rodríguez González, Vicente; Pérez Larios, Alejandro y Li Puma, Gianluca (eds.). (2013). Photocatalysis: From the treatment of emerging contaminats to energy conversión Journal of Hazardous Materials, vol. 263, parte 1, diciembre 15.<http://dx.doi.org/10.1016/j.jhazmat.2013.11.001> <http://www.sciencedirect.com/science/journal/03043894/263/part/P1〉

3. Número especial:

Rodríguez González, Vicente; Hernández Gordillo, Agileo y Hang Hu, Yan (eds.). (2016). Novel nanomaterials for photocatalysis, photochemistry, and photobiology. Catalysis Today, vol. 266, 1-226, mayo 15, <http://dx.doi.org/10.1016/j. cattod.2016.02.012〉

<http://www.sciencedirect.com/science/journal/09205861/266>.

4. Rodríguez González, Vicente; Zanella Specia, Rodolfo; Hinojosa Reyes, Mariana (eds. responsables). (2014). Abstract Book from the 3rd Latin-American Congress of Photocatalysis, Photochemistry and Photobiology. San Luis Potosí-IPICyT, octubre 14-17.

5. 〈https://sites.google.com/a/ipicyt.edu.mx/vicenterodriguez/>

6. <http://www.ipicyt.edu.mx/Materiales_Avanzados/areas_materiales_avanzados. $\mathrm{php}>$ 
Mundo Nano | CATÁLISIS EN MÉXICo | www.mundonano.unam.mx

10(18), enero-junio 2017 | DOI: 10.22201/ceiich.24485691e.2017.18.60108

7. Rodríguez González, Vicente; Li Puma, Gianluca; Dionysios, Dionysiou y Rodolfo Zanella Specia. (2015). Special Section on Photoassisted IPICyT-2014. Journal of Environmental Chemical Engineering, vol. 3, núm. 4, parte B, 3037-3062 pp., diciembre. 